\title{
Diacronie
}

Studi di Storia Contemporanea

$\mathrm{N}^{\circ} 28,4 \mid 2016$

La voce del silenzio

\section{Diarmaid Ferriter, A Nation and not a Rabble, The Irish Revolution, 1913-1923}

\section{Cecilia Biaggi}

\section{Q OpenEdition \\ 1 Journals}

Edizione digitale

URL: http://journals.openedition.org/diacronie/4556

DOI: $10.4000 /$ diacronie.4556

ISSN: 2038-0925

Editore

Association culturelle Diacronie

Notizia bibliografica digitale

Cecilia Biaggi, « Diarmaid Ferriter, A Nation and not a Rabble, The Irish Revolution, 1913-1923 », Diacronie [Online], N²8, 4 | 2016, Messo online il 29 décembre 2016, consultato il 24 septembre 2020. URL : http://journals.openedition.org/diacronie/4556 ; DOI : https://doi.org/10.4000/diacronie.4556 


\title{
Diacronie
}

N. 28 | 4|2016 La voce del silenzio: intelligence, spionaggio e conflitto nel XX secolo

\section{PANORAMICA: Irlanda 2015}

\author{
Cecilia BIAGGI*
}

FERRITER, Diarmaid, A Nation and not a Rabble, The Irish Revolution, 1913-1923, Profile Books, London, 2015, 528 pp.

A Nation and not a Rabble ("una nazione e non una marmaglia") ha riscosso molto successo in un'Irlanda entrata tre anni fa nella "Decade of Commemorations" (Decade delle Commemorazioni). Il libro tratta appunto della Rivoluzione irlandese, iniziata nel 1913 con "la serrata di Dublino", e terminata con la fine della guerra civile nel 1923.

Ferriter si propone di contribuire ad una storia della Rivoluzione "dal basso" che indaghi le diverse istanze di cui si fecero portatori coloro che vi parteciparono, mettendo in discussione l'opinione diffusa secondo cui la nazione irlandese si sollevò contro l'oppressione inglese senza distinzioni di classe, sesso, età. Il libro presenta un'approfondita e bilanciata analisi sociale dell'Irlanda di inizio Novecento, un periodo di pace e stabilità in cui Ferriter individua tuttavia le origini del malcontento che portò alla Rivoluzione.

Nonostante la trattazione sembri inizialmente vertere attorno agli antitetici concetti di nation e rabble, nel corso della narrazione essi vengono ripetutamente abbandonati e, infine, rimangono vaghi e sfuggenti. Si può concludere che la rabble sia costituita da tutti coloro che per diverse ragioni non facevano parte della nazione irlandese. Tuttavia, nonostante il termine venga spesso usato per indicare il proletariato, l'analisi di Ferriter non è sufficiente a definire la rabble soltanto in termini di classe. Ciò che emerge chiaramente sono invece timore e disprezzo per la rabble da parte della classe politica, a cui il titolo si riferisce. Nel gennaio 1922, all'indomani della nascita dello Stato Libero d'Irlanda, George Gavan Duffy, Ministro degli Esteri, espresse la preoccupazione che il crescente antagonismo tra sostenitori ed oppositori dell'accordo con la Gran Bretagna 
presentasse il paese agli stranieri non come una nazione ma, appunto, come una marmaglia.

Il successo di questo libro è dovuto anche alla notorietà di Ferriter: Professore di Storia irlandese moderna e contemporanea all'University College Dublin, è spesso ospite alla radio e alla televisione nazionale. Inoltre, è parte di un gruppo di undici esperti che collaborano con il governo irlandese nell'organizzazione delle celebrazioni in memoria della Pasqua di Sangue del 1916.

Non stupisce dunque che una parte consistente di $A$ Nation and not a Rabble sia dedicata alla storiografia della Rivoluzione e alla sua commemorazione. Tuttavia, la discussione della letteratura e delle fonti, contenente dettagliate informazioni sulla loro accessibilità dagli anni Cinquanta ad oggi, risulterà probabilmente eccessiva e in parte ostica ad un pubblico di non addetti ai lavori. Al contrario, l'acceso dibattito sull'eredità e commemorazione della Rivoluzione, che occupa quasi 200 pagine, è di stimolante attualità.

FLANAGAN, Frances, Remembering the Revolution: Dissent, Culture, and Nationalism in the Irish Free State, Oxford, Oxford University Press, 2015, 272 pp.

Come molte delle opere a carattere storico pubblicate negli ultimi anni in Irlanda, Remembering the Revolution, affronta uno dei temi più controversi della storia nazionale, ovvero la lotta per l'indipendenza iniziata nel 1916. Flanagan si concentra su come quegli eventi vennero ricordati nei due decenni successivi, contrapponendo alla celebrazione della Rivoluzione da parte dei diversi governi in carica, il dissenso di quattro intellettuali dell'epoca: Eimar O’Duffy, P.S. O’Hegarty, George Russell, e Desmond Ryan. Nonostante le diversità ideologiche, e le loro personali esperienze della Rivoluzione, costoro furono fortemente disillusi dalla guerra civile e in seguito non riconobbero nello Stato Libero gli ideali per cui gli irlandesi avevano combattuto. Fortemente critici nei confronti dell'ingerenza clericale nella politica interna, essi si avvicinarono al socialismo, mentre venivano accusati di tradimento per le loro posizioni critiche nei confronti della Rivoluzione e delle sue conquiste. Ripercorrendo le vite di questi quattro intellettuali, generalmente sottovalutati e - soprattutto Ryan e O’Duffy - quasi ignorati dalla storiografia, Flanagan contribuisce al dibattito storiografico sulla Rivoluzione delineando le origini di quel dissenso che, decenni più tardi, diede origine al revisionismo. 


\section{FANNING, Ronan, Éamon de Valera: A Will to Power, London, Faber \&}

\section{Faber, 2015, 320 pp.}

In questa lucida biografia, Fanning descrive de Valera come il personaggio più controverso e dibattuto della storia irlandese, di cui fu protagonista dall'Insurrezione di Pasqua del 1916 fino al 1973 quando, novantenne, si ritirò dalla vita pubblica. De Valera fu ripetutamente Primo Ministro e poi Presidente della Repubblica, redasse la Costituzione del 1937 e fondò uno dei due principali partiti politici irlandesi, Fianna Fáil.

Uno dei misteri irrisolti che circondano de Valera rimane il suo rifiuto di guidare la delegazione irlandese che nel 1921 si recò a Londra per negoziare la fine delle ostilità tra IRA e Gran Bretagna. Fanning analizza nel dettaglio la teoria di associazione esterna tra Irlanda e Gran Bretagna formulata da de Valera, concludendo che egli non riuscì a farla comprendere appieno ai suoi colleghi. Di conseguenza la scelta di non partecipare ai negoziati risulta, ancora una volta, incomprensibile. Tuttavia Fanning non esita a condannare le azioni di de Valera in seguito alla firma del trattato che diede vita allo Stato Libero, invece della tanto agognata repubblica. De Valera rifiutò il trattato, causando una frattura che in pochi mesi sfociò nella guerra civile.

Nonostante l'importanza di questi eventi, Fanning dedica altrettanto spazio al ruolo di de Valera nei decenni successivi, concludendo che egli riuscì a garantire al paese una stabilità politica sconosciuta a molti stati europei nati alla fine della prima guerra mondiale. Tuttavia, il prezzo da pagare fu una politica conservatrice responsabile della stagnazione economica e dell'emigrazione.

\section{WALSH, Maurice, Bitter Freedom: Ireland in a Revolutionary World 1918- 1923, London, Faber \& Faber, 2015, 544 pp.}

L'ultima opera di Walsh si distingue per lo stile semplice della narrazione, vivacizzata da aneddoti e curiosità. Inoltre, si distanzia dalla visione dominante della storia irlandese come "eccezionale", che pone l'Irlanda al di fuori del contesto internazionale per concentrarsi sulle sue dinamiche interne e sulla relazione con la Gran Bretagna. Negli ultimi anni ci sono stati timidi tentativi di interpretare l'Irlanda come una componente essenziale dell'Impero Britannico, e si è cominciato, per esempio, a confrontare l'amministrazione dell'isola con quella coloniale dell'India. Tuttavia, la maggior parte degli studi di storia irlandese rimane nel solco della tradizione, e dunque Bitter Freedom rappresenta una significativa eccezione. 
Diversi leader rivoluzionari irlandesi cercarono, con scarso successo, di legittimare le loro istanze inserendole in un contesto Wilsoniano di lotta di liberazione di una nazione oppressa. Se la rappresentanza repubblicana al congresso di pace di Parigi fu un fallimento, la campagna di raccolta fondi negli Stati Uniti si rivelò molto efficace, anche se il sostegno che gli irlandesi ricevettero da alcuni leader afroamericani non fu sempre bene accolto.

Il contributo di Walsh in termini di fonti primarie non è particolarmente rilevante in quanto limitato a memorie personali e testimonianze di osservatori internazionali. Egli riesce comunque ad evidenziare in modo incisivo sia le contraddizioni ideologiche dei principali attori della Rivoluzione, sia l'impatto che questa ebbe sulle vite quotidiane dei semplici spettatori. 


\section{* Gli autori}

Cecilia Biaggi sta concludendo un dottorato di ricerca in Storia presso l'Università di Oxford. La sua tesi si occupa di forme di partecipazione politica non tradizionali in Irlanda del Nord tra il 1920 ed il 1932. In precedenza, ha passato un anno al Trinity College di Dublino come studente Erasmus, dove ha svolto ricerca per la tesi di laurea magistrale sui Troubles, conseguita presso l'Università di Siena nel 2011. Ha conseguito la laurea triennale nel 2008 all'Università di Bologna.

URL: < http://www.studistorici.com/progett/autori/\# Biaggi >

\section{Per citare questo articolo:}

BIAGGI, Cecilia, «Panoramica: Irlanda 2015», Diacronie. Studi di Storia Contemporanea : La voce del silenzio: intelligence, spionaggio e conflitto nel XX secolo, 29/12/2016,

URL:< http://www.studistorici.com/2016/12/29/irlanda_numero_28/ >

Diacronie Studi di Storia Contemporanea $\beta$ www.diacronie.it

Risorsa digitale indipendente a carattere storiografico. Uscita trimestrale. redazione.diacronie@hotmail.it

Comitato di redazione: Jacopo Bassi - Luca Bufarale - Antonio César Moreno Cantano - Deborah Paci - Fausto Pietrancosta - Alessandro Salvador - Matteo Tomasoni - Luca Zuccolo

Diritti: gli articoli di Diacronie. Studi di Storia Contemporanea sono pubblicati sotto licenza Creative Commons 3.0. Possono essere riprodotti e modificati a patto di indicare eventuali modifiche dei contenuti, di riconoscere la paternità dell'opera e di condividerla allo stesso modo. La citazione di estratti è comunque sempre autorizzata, nei limiti previsti dalla legge. 